\section{Clinical Utility of Antipeptidyl Arginine Deiminase Type 4 Antibodies}

\section{To the Editor:}

Rheumatoid factor (RF) and anticitrullinated protein antibodies (ACPA) are important biomarkers in the diagnosis of rheumatoid arthritis (RA) but leave a gap of $>50 \%$ seronegative in early $\mathrm{RA}^{1}$. In addition, there is marked clinical heterogeneity in the seropositive group, precluding the use of RF and ACPA alone as prognostic biomarkers. These characteristics drive the demand for novel diagnostic and prognostic markers in $\mathrm{RA}^{1}$. In this context, we read the recent paper by Guderud, et al on the clinical utility of antipeptidyl arginine deiminase type 4 (anti-PAD4) antibodies with great interest ${ }^{2}$. The authors studied anti-PAD4 antibodies in 745 patients with RA using a dissociation-enhanced lanthanide fluorescence immunoassay (DELFIA) and found $26 \%$ to be positive. In addition, the study also investigated the genotype of PADI4 using TaqMan assays in 945 patients and 1118 controls. Based on the results, the authors concluded that anti-PAD4 antibodies are not useful clinical biomarkers in RA. Unfortunately, there are some significant questions and concerns regarding this conclusion.

Importantly, the authors did not specify what they meant by "no current clinical utility in RA," and their conclusion was based on a limited clinical dataset composed of disease activity measures (modified Health Assessment Questionnaire and 28-joint count Disease Activity Score), swollen and tender joint counts, and systemic markers of acute inflammation (C-reactive protein and erythrocyte sedimentation rate). Clinical utility is a broad term that may apply to many aspects of the journey of a patient with RA, including disease prediction and prevention ${ }^{3}$, diagnosis, stratification, prognosis, and monitoring. Therefore, care needs to be taken in declaring that a putative biomarker has no clinical utility without addressing this range of potential applications.

This is especially true in the case of anti-PAD4 antibodies, which have been reproducibly shown to be associated with radiographic joint damage in 4 independent cohorts of patients with RA (Table 1) ${ }^{4,5}$. Importantly, these and other cohorts have revealed variable associations of anti-PAD4 antibodies with acute measures of inflammation and disease activity scores, such as those measured by Guderud, et $a l^{2}$. Thus, the findings of this current manuscript are consistent with the published literature but failed to determine the association of anti-PAD4 antibodies with joint damage, the singular clinical aspect of RA that has been reproducibly associated with this putative biomarker. These factors suggest that although anti-PAD4 antibodies may be of questionable utility in measuring acute inflammation in RA (a feature readily measured by existing laboratory tests and physician examination), they may have clinical value as a prognostic biomarker related to the accumulation of radiographic joint damage ${ }^{5,6,7,8}$.

Regarding the potential utility of anti-PAD4 antibodies in the diagnosis of RA, Guderud, et al report that $6 \%$ of the total RA patient population is anti-PAD4+/ACPA- . This equates to $15.8 \%$ of the ACPA- subgroup and is consistent with the 2-17.7\% PAD4+/ACPA- patients described in other RA cohorts. Further, 2 recent review articles analyzed the prevalence and performance characteristics of anti-PAD4 antibodies ${ }^{9,10}$. Both were consistent in their conclusion that anti-PAD4 antibodies showed good discrimination between RA and controls, and the more recent article presents a metaanalysis ${ }^{10}$ with a pooled sensitivity and specificity of anti-PAD4 antibodies for RA of $38.0 \%$ (95\% CI 30.0-46.0\%) and $96.0 \%$ (95\% CI 93.0-98.0\%), respectively. This suggests that anti-PAD4 antibodies may also be clinically useful for RA diagnosis even within traditionally seronegative individuals.

This recent report also raises several questions regarding the method used to detect anti-PAD4 antibodies and the need for consistency in the field. First, the DELFIA method used by Guderud, et al is not a validated technology for the detection of autoantibodies, has not been compared directly to ELISA or immunoprecipitation for the detection of anti-PAD4 antibodies, and does not play a significant role in diagnostic settings. In addition, it is unclear which controls have been included to assess the performance of the test, and no information is provided regarding batch effects, standardization, or calibration. This is particularly important because the authors combined data from 2 different cohorts, in which antibody testing was performed at drastically different timepoints, and came to markedly different conclusions. Of the total subjects, 366 of 745 patients were from a study by Halvorsen, et $a l^{5}$ from 2008 that was among the first to determine that anti-PAD4 antibodies are associated with markers of disease severity, including radiographic joint damage, in patients with RA (Table 1).

The conclusion that anti-PAD4 antibodies are not clinically useful is not supported by the data presented by Guderud, et $a l^{2}$ and contrasts with a body of published literature. We acknowledge that anti-PAD4 antibodies might not have diagnostic utility when compared to ACPA, but they hold clinical promise in assessing disease prognosis as it relates to the accumulation of radiographic joint damage and in disease diagnosis of RA, especially in ACPA-negative individuals.

Table 1. Summary of studies reporting the prognostic value of anti-PAD4 antibodies in RA.

\begin{tabular}{|c|c|c|c|c|c|c|c|c|c|c|c|}
\hline \multirow[b]{2}{*}{ Study } & \multirow[b]{2}{*}{ Cohort } & \multirow[b]{2}{*}{$\begin{array}{c}\text { Anti-PAD4 } \\
\text { Assay }\end{array}$} & \multirow[b]{2}{*}{ Anti-CCP } & \multicolumn{5}{|c|}{ Association with Anti-PAD4 Antibodies } & \multirow[b]{2}{*}{ CRP } & \multirow[b]{2}{*}{ ESR } & \multirow[b]{2}{*}{ Joint Damage } \\
\hline & & & & Duration & DAS28 & HAQ & SJC & TJC & & & \\
\hline \multirow[t]{2}{*}{ Guderud 2018} & EURIDISS & DELFIA & NR & NR & NR & NR & NR & NR & NR & NR & NR \\
\hline & ORAR & DELFIA & + & + & \pm & - & \pm & - & \pm & - & NR \\
\hline Navarro-Millan 2016 & CLEAR & IP & + & + & NR & NR & NR & NR & - & NR & + \\
\hline Reyes-Castillo 2015 & JMR & ELISA & + & + & - & - & NR & NR & - & - & NR \\
\hline Darrah 2013 & ESCAPE & IP & + & + & - & - & NR & NR & - & NR & + \\
\hline Kolfenbach 2010 & Pre-RA & IP & + & NR & NR & NR & NR & NR & NR & NR & NR \\
\hline Harris 2008 & ESCAPE & IP & + & + & NR & NR & NR & NR & NR & NR & + \\
\hline \multirow[t]{2}{*}{ Halvorsen 2008} & EURIDISS & ELISA & + & + & NR & NR & NR & NR & + & - & NR \\
\hline & ORAR & ELISA & + & + & & + & + & - & + & - & + \\
\hline Zhao 2008 & PUPH & ELISA & + & NR & + & NR & NR & NR & - & + & + \\
\hline
\end{tabular}

+: yes; -: no; \pm : associated in univariate but not multivariate models. RA: rheumatoid arthritis; anti-CCP: anticyclic citrullinated peptide antibodies; DAS28: 28-joint count Disease Activity Score; HAQ: Health Assessment Questionnaire; SJC: swollen joint count; TJC: tender joint count; CRP: C-reactive protein; ESR: erythrocyte sedimentation rate; DELFIA: dissociation-enhanced lanthanide fluorescence immunoassay; EURIDISS: European Research on Incapacitating Disease and Social Support; ORAR: Oslo RA Register; CLEAR: Consortium for the Longitudinal Evaluation of African Americans with Early Rheumatoid Arthritis; JMR: Jalisco, Mexico Rheumatology; ESCAPE: Evaluation of Subclinical Cardiovascular Disease and Predictors of Events in Rheumatoid Arthritis; PUPH: Peking University People's Hospital; NR: not reported; anti-PAD4: antipeptidyl arginine deiminase type 4; IP: immunoprecipitation.

Personal non-commercial use only. The Journal of Rheumatology Copyright @ 2019 . All rights reserved. 
ERIKA DARRAH, ED, PhD, Division of Rheumatology, Johns Hopkins University School of Medicine, Baltimore, Maryland; LAURA MARTINEZ-PRAT (D), LM-P, BS, Research and Development, Inova Diagnostics Inc.; MICHAEL MAHLER, MM, PhD, Research and Development, Inova Diagnostics Inc., San Diego, California, USA. E. Darrah is an author on licensed patent no. 8,975,033 entitled "Human Autoantibodies Specific for PAD3 which are Cross-reactive with PAD4 and their Use in the Diagnosis and Treatment of Rheumatoid Arthritis and Related Diseases." Address correspondence to M. Mahler, Inova Diagnostics, 9900 Old Grove Road, San Diego, California 32131-1638, USA.E-mail: mmahler@inovadx.com or m.mahler.job@web.de

\section{REFERENCES}

1. Trouw LA, Mahler M. Closing the serological gap: promising novel biomarkers for the early diagnosis of rheumatoid arthritis. Autoimmun Rev 2012;12:318-22.

2. Guderud K, Mæhlen MT, Nordang GB, Viken MK, Andreassen BK, Molberg $\varnothing$, et al. Lack of association among peptidyl arginine deiminase type 4 autoantibodies, PADI4 polymorphisms, and clinical characteristics in rheumatoid arthritis. J Rheumatol 2018;45:1211-19.

3. Mahler M. Population-based screening for ACPAs: a step in the pathway to the prevention of rheumatoid arthritis? Ann Rheum Dis 2017;76:e42.

4. Darrah E, Giles JT, Ols ML, Bull HG, Andrade F, Rosen A. Erosive rheumatoid arthritis is associated with antibodies that activate PAD4 by increasing calcium sensitivity. Sci Transl Med 2013;5:186ra65.

5. Halvorsen EH, Pollmann S, Gilboe IM, van der Heijde D, Landewe
R, Odegard S, et al. Serum IgG antibodies to peptidylarginine deiminase 4 in rheumatoid arthritis and associations with disease severity. Ann Rheum Dis 2008;67:414-7.

6. Zhao J, Zhao Y, He J, Jia R, Li Z. Prevalence and significance of anti-peptidylarginine deiminase 4 antibodies in rheumatoid arthritis. J Rheumatol 2008;35:969-74.

7. Harris ML, Darrah E, Lam GK, Bartlett SJ, Giles JT, Grant AV, et al. Association of autoimmunity to peptidyl arginine deiminase type 4 with genotype and disease severity in rheumatoid arthritis. Arthritis Rheum 2008;58:1958-67.

8. Navarro-Millan I, Darrah E, Westfall AO, Mikuls TR, Reynolds RJ, Danila MI, et al. Association of anti-peptidyl arginine deiminase antibodies with radiographic severity of rheumatoid arthritis in African Americans. Arthritis Res Ther 2016;18:241.

9. Reyes-Castillo Z, Palafox-Sanchez CA, Parra-Rojas I, Martinez-Bonilla GE, del Toro-Arreola S, Ramirez-Duenas MG, et al. Comparative analysis of autoantibodies targeting peptidylarginine deiminase type 4 , mutated citrullinated vimentin and cyclic citrullinated peptides in rheumatoid arthritis: associations with cytokine profiles, clinical and genetic features. Clin Exp Immunol 2015;182:119-31.

10. Ren J, Sun L, Zhao J. Meta-analysis: diagnostic accuracy of antibody against peptidylarginine deiminase 4 by ELISA for rheumatoid arthritis. Clin Rheumatol 2017;36:2431-8.

First Release January 15 2019; J Rheumatol 2019;46:3; doi:10.3899/jrheum180905 